Ebisu Ebisu

Études japonaises Études japonaises

49 | printemps-été 2013

De chose en fait : la question du milieu

\title{
Tétralemme et milieu humain : la mésologie à la lumière de Yamauchi
}

Tetralemma and Human Milieu: Mesology in the Light of Yamauchi

テトラレンマと風土一山内得立著『ロゴスとレンマ』の光に照らし

た風土論一

\section{Augustin Berque}

\section{OpenEdition}

Journals

Édition électronique

URL : http://journals.openedition.org/ebisu/731

DOI : 10.4000/ebisu.731

ISSN : 2189-1893

Éditeur :

Institut français de recherche sur le Japon (UMIFRE 19 MAEE-CNRS), Maison franco-japonaise

Édition imprimée

Date de publication : 1 avril 2013

Pagination : $57-71$

ISSN : 1340-3656

\section{Référence électronique}

Augustin Berque, «Tétralemme et milieu humain : la mésologie à la lumière de Yamauchi », Ebisu [En ligne], 49 | printemps-été 2013, mis en ligne le 04 avril 2014, consulté le 30 avril 2019. URL : http:// journals.openedition.org/ebisu/731 ; DOI : 10.4000/ebisu.731 


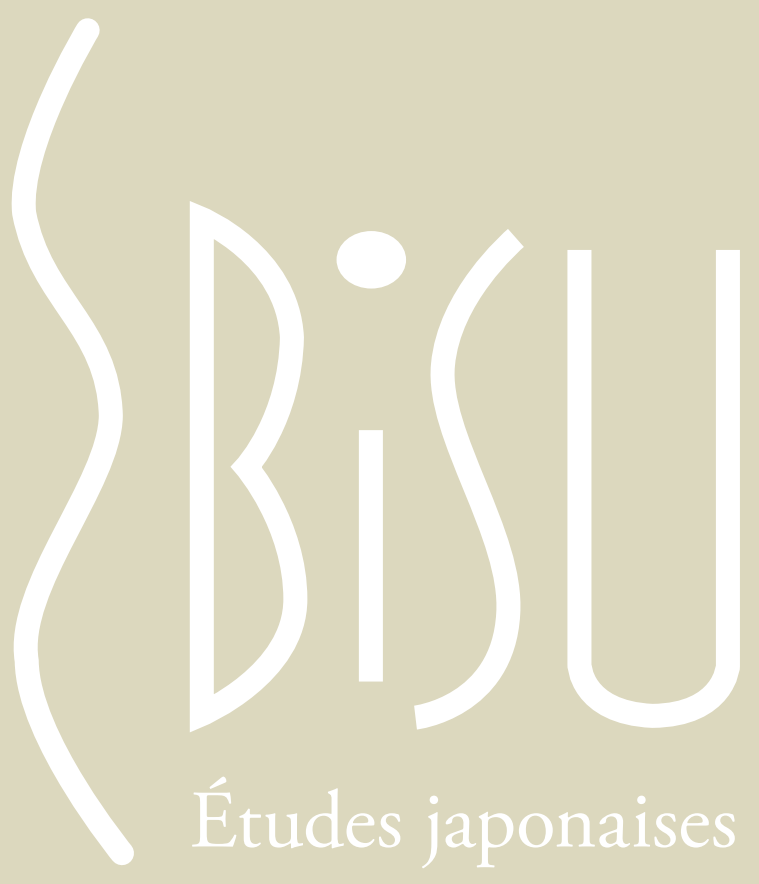

Dossier

De chose en fait : la question du milieu

Articles issus du colloque de Shin-Hirayu

Coordonné par Augustin BERQue

Varia | Nicolas Baumert - Peut-il exister des terroirs du saké ?

Conférences de la MFJ | François Macé - Le Kojiki, une Énéide longtemps oubliée ? | Patrick Beillevaire - Présences françaises à Okinawa : de Forcade (1844-1846) à Haguenauer (1930)

Livres à lire

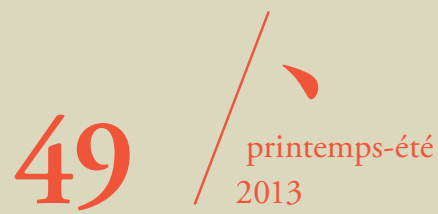




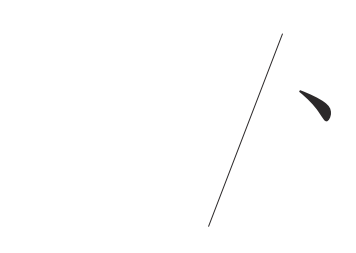

\section{Dossier}

\section{De chose en fait : la question du milieu}

Mono kara koto e to füdo no mondai

物から事へと風土の問題

Articles issus $d u$

colloque de Shin-Hirayu

Dossier coordonné

par Augustin Berque 


\section{Tétralemme et milieu humain La mésologie à la lumière de Yamauchi}

Augustin BERQUE*

\section{1. À l'origine de la problématique des milieux}

La question des milieux n'a explicitement été problématisée comme telle que le 7 juin 1848, dans un exposé de Charles Philippe Robin (1821-1885, médecin, homme de science et homme politique français) à la séance inaugurale de la Société de biologie intitulée Sur la direction que se sont proposée en se réunissant les membres fondateurs de la Société de biologie pour répondre au titre quils ont choisi.

Robin y exposait la classification comtienne des sciences, y traitait, dans l'esprit du Cours, des tâches de la biologie, au premier rang desquelles la constitution d'une étude des milieux, pour laquelle Robin inventait même le terme de mésologie. (Canguilhem 2002: 72).

Si toutefois la notion de mésologie est ainsi datée, et si son objet, la notion de milieu, n'est comme on le verra pas beaucoup plus ancienne, en revanche la question posée remonte à peu près aussi loin que l'ontologie dans la pensée européenne. En effet, si l'on définit cette question comme celle du rapport entre les êtres et ce qui les entoure, on la trouve déjà dans le Timée à propos de la chôra $(\chi \dot{\omega} p \alpha)^{1}$. Le sens fondamental de ce terme chôra,

* EHESS

1. Sur ce thème, voir Berque 2012. 
c'est celui de territoire approprié par une polis (cité-État), dont il permet l'existence. C'est donc un rapport d'adéquation mutuelle. Concrètement, la chôra, c'est d'abord la campagne qui entoure la ville (astu), et qui la nourrit. Platon reprend métaphoriquement ces réalités en termes ontologiques, faisant de la chôra la nourrice (tithênê) de l'être relatif, appelé genesis, c'est-à-dire l'existant, qui naît (d'où le terme genesis), vit et disparaît. À la différence de l'être absolu (on, idea, eidos), dont l'existant n'est que le reflet, et qui existe en lui-même, hors du temps et de l'espace, la genesis ne peut pas exister sans une chôra. Son être en est donc inséparable, relation qui est ambivalente ; car la chôra n'est pas seulement la nourrice et même la mère (mêtêr) de l'existant, elle en est aussi l'empreinte (ekmageion) ${ }^{2}$.

Vis-à-vis de l'existant, la chôra - autrement dit le milieu ${ }^{3}$ de cet existant est ainsi à la fois une chose (une empreinte) et son contraire (une matrice) ; donc à la fois $A$ et $\bar{A}$ (non-A). C'est là une aporie que Platon ne surmonte pas. Il ne définit donc pas la chôra, se contenant de la cerner par diverses métaphores comme celles ci-dessus, et laissant irrésolue la contradiction entre l'empreinte et la matrice. J'ai récemment avancé l'hypothèse (Berque 2012) que cette aporie vient de ce que le rationalisme platonicien reposant sur le principe du tiers exclu, à savoir qu'il ne peut y avoir de tiers terme qui soit à la fois $\mathrm{A}$ et $\bar{A}$. Platon ne pouvait admettre intellectuellement le " troisième et autre genre " (triton allo genos) - ni l'être absolu, ni l'être relatif - qu'il attribue néanmoins à la chôra.

J'entends ici poursuivre cette hypothèse en montrant que ce " troisième genre " est bel et bien celui de la réalité des milieux, et que la pensée de Yamauchi Tokuryū 山内得立 (1890-1982), telle que celui-ci l'expose dans Rogosu to Renma ロゴスとレンマ (Logos et Lemme, 1974), nous permet aujourd'hui de concevoir une lemmique - une logique des prises (lêmmata $\lambda \dot{\eta} \mu \mu \alpha \tau \alpha$, de lambanô $\lambda \alpha \mu \beta \dot{\alpha} \nu \omega$, prendre; d'où lemme) que nous entretenons avec la réalité des choses concrètes de notre milieu, par contraste avec l'abstraction de la logique formelle ; lemmique susceptible de dépasser enfin rationnellement les apories que nous ont léguées le principe du tiers exclu et son avatar ontologique, le dualisme moderne, sans pour autant sombrer dans l'illusion qu'il suffisait de culbuter la logique de l'identité du sujet en

2. Pour plus de détails sur ces questions, voir Berque 2009, Chap. I.

3. Je me rallie ici à l'interprétation de Brisson (1994: 175 sqq.). 
son énantiomère, la logique du prédicat (jutsugo no ronri 述語の論理), ou " logique du lieu » (basho no ronri 場所の論理), pour réaliser le « dépassement de la modernité » (kindai no chökoku 近代の超克), comme a pu le faire naguère l'École de Kyoto (Kyōto gakuha 京都学派) ${ }^{4}$, autour de Nishida Kitarō 西田幾多郎 (1870-1945).

\section{La distinction entre milieu et environnement}

Au sens indiqué plus haut - le rapport entre les êtres et ce qui les entoure, la notion de milieu date de la seconde moitié du XvIII ${ }^{\mathrm{e}}$ siècle. Georges Canguilhem, dans La Connaissance de la vie, en a détaillé l'histoire (Canguilhem 2009, Chap. III). Cette notion est alors dérivée de la mécanique, où elle existait déjà mais sous un autre nom. Newton emploie à cet égard le terme de fluid, dont l'éther constitue le type. Le Concise Oxford Dictionary (éd. 1964) définit ainsi le sens physique d'ether : "A medium assumed to permeate space $\&$ fill interstices between particles of air and other matter, medium in which electro-magnetic waves are transmitted. »

Pour Newton, il s'agissait en effet d'expliquer comment pouvait s'exercer l'action à distance entre deux corps. Canguilhem montre que, pour Descartes, ce problème ne se posait pas ; car dans le cadre de sa physique, l'action entre les corps ne pouvait s'exercer que par contact direct, sans l'intermédiaire d'un tiers terme. On notera que cette conception cartésienne est fondamentalement dérivée du dualisme, lequel a finalement expulsé l'idée d'éther hors de la physique.

Dans les sciences du vivant et les sciences humaines toutefois, la chose n'a pas été aussi simple. Avec Auguste Comte, le milieu acquiert le statut d'un principe universel et abstrait de l'explication en biologie. Beaucoup de naturalistes de cette époque concevront en termes de réciprocité la relation entre l'organisme et son milieu (ici entendu au sens d'environnement, terme peu utilisé jusque vers 1970), comme déjà la voyait Comte, pour qui l'organisme est approprié à son milieu, et le milieu favorable à l'organisme (Canguilhem 2009 : 170). Toutefois, le courant général de la pensée dérivera vers un déterminisme où c'est le milieu qui exerce, causalement, une

4. Sur ce thème, voir Berque 2000, et plus particulièrement Berque 2002. 
action sur l'organisme. Cette vision dominera notamment la géographie allemande ; d'où la notion de " déterminisme géographique ».

Le " possibilisme » de l'école française de géographie devait plus tard montrer les limites de cette conception, avec notamment La Terre et l'évolution humaine, de l'historien Lucien Febvre (1922). Toutefois, si c'était là reconnaître le libre arbitre du sujet humain, l'on restait dans le cadre du dualisme. L'accent mis sur la contingence par le possibilisme ne constituait pas un principe explicatif, et même, en un sens, éludait au contraire le problème central posé dès l'origine par la notion de milieu ; tendance qui devait mener les sciences humaines à ne plus voir la question qu'en termes de projections arbitraires du sujet sur l'objet.

C'est alors que, dans les sciences naturelles comme dans les sciences humaines, un changement radical de point vue se manifesta. Le naturaliste allemand Jakob von Uexküll (1864-1944), en 1934, résume dans un petit livre les idées principales de ses longues recherches sur la voie de ce qui allait devenir l'éthologie. Intitulé Streifzüge durch die Umwelten von Tieren und Menschen (Incursions dans les milieux animaux et humain), ce livre sera traduit en français sous le titre Mondes animaux et monde humain (1965), puis retraduit sous celui de Milieu animal et milieu humain (2010). Uexküll y introduit une distinction révolutionnaire entre Umgebung (le donné environnemental objectif) et Umwelt (le milieu, ou le monde ambiant, propre à une espèce donnée, tel que celle-ci le perçoit). Corrélativement, Uexküll introduit toute une série de concepts, tels Merkbild (image-perception), Suchbild (image-prospection), Wirkbild (image-action), Merkmal (signe perceptif), Fresston (tonalité de nutrition), Wohnton (tonalité d'habitat), etc. L'idée générale est que l'espèce et son milieu sont une élaboration réciproque, au sein de laquelle l'animal n'est pas une machine réagissant à des actions par des mouvements, mais plutôt un machiniste réagissant à des signaux par des opérations.

C'est là clairement dépasser le dualisme. La réalité du milieu (Umwelt) est au-delà de la dichotomie sujet-objet. C'est un tiers genre, un triton allo genos insaisissable par le mécanicisme moderne et la logique du tiers exclu qui le sous-tend. Cependant, Uexküll n'en a pas lui-même tiré les 
conclusions logiques et ontologiques ; sa pensée, du moins, a influencé des philosophes comme Heidegger, Deleuze, Agamben et d'autres ${ }^{5}$.

Il n'est pas impossible que, durant son séjour en Allemagne en 1927, Watsuji Tetsurō 和辻哲郎 (1889-1960) ait entendu parler d'Uexküll, mais on n'en a aucune trace. C'est explicitement en réaction à Heidegger que le philosophe japonais, à partir de 1928, commence à publier une série d'articles qu'il rassemblera en 1935 dans un livre : Füdo. Ningengakuteki kōsatsu 風土一人間学的考察 (Milieux. Études de l'entrelien humain) ${ }^{6}$. Dans ce livre, Watsuji introduit une distinction fondatrice entre kankyo 環境 (l'environnement, tel qu'il est objectivé par la science) et füdo 風土 (le milieu, tel qu'il est concrètement vécu par une certaine société). Cette distinction est exactement homologue à celle établie par Uexküll entre Umgebung et Umwelt, à ceci près que le propos d'Uexküll se situe au niveau ontologique du vivant en général, et le propos de Watsuji à celui de l'humain en particulier.

\section{Médiance et trajection}

Corrélativement, Watsuji introduit le concept ontologique de füdosei 風 土性, qu'il définit, à la première ligne de l'ouvrage, comme " le moment structurel de l'existence humaine" (ningen sonzai no kōzō keiki 人間存在の 構造契機). Ce concept a été rendu en français par médiance (Berque 1986), néologisme dérivé du latin medietas, qui veut dire "moitié ". Lidée de Watsuji est en effet que, dans l'humain, se combinent dynamiquement (i.e. en un " moment ", comme deux forces en mécanique) deux " moitiés ", l'une individuelle, le hito 人, et l'autre commune, l'aida 間, entrelien qui se tisse entre les personnes et avec les choses, constituant historiquement un certain milieu. De cette combinaison entre l'individu et son milieu émerge l'humain, ningen 人間. Ainsi, l'humain est nécessairement médial (füdoteki 風土的).

5. Sur l'influence d'Uexküll sur Heidegger (qui lui consacra un séminaire entier), voir Agamben (2002).

6. Watsuji 2011. Les versions anglaise et allemande de Füdo n'ont pas traduit ce concept. La traduction espagnole le rend par ambientalidad. 
Avec Watsuji, l'approche théorique de la médiance est initialement celle de la phénoménologie herméneutique. Vue d'aujourd'hui, l'homologie susdite avec l'approche d'Uexküll lui donne néanmoins d'emblée un fondement dans les sciences de la nature, lequel, des progrès de l'éthologie à la récente révolution post-génomique, n’a depuis cessé de se renforcer. La biologie en effet, dans la formation du vivant à tous les niveaux, accorde aujourd'hui un rôle croissant à l'épigénétique, c'est-à-dire aux processus d'interaction avec le milieu (Boi 2008). Du côté des sciences humaines, sans parler de la phénoménologie (en particulier de la mise en évidence, par Merleau-Ponty [1945], de la corporéité), le couplage entre " corps animal " (individuel) et " corps social " (commun), dont Leroi-Gourhan (1964) - sur des bases positivistes sans rapport avec la phénoménologie - a fait le moteur de l'émergence de notre espèce, n'est autre que la médiance. Plus près de nous, en reliant une très ancienne idée (celle selon laquelle l'humain est un être inachevé) à la notion biologique de néoténie (i.e. en l'occurrence que l'humain, à sa naissance, est un prématuré), Dany-Robert Dufour (2005) a montré qu'il en résulte structurellement, chez l'individu, le besoin de s'aliéner à un Autre qui le complète, notamment à ce que les monothéismes ont appelé Dieu ; couplage qui, derechef, n’est autre que la médiance.

À défaut du concept lui-même, ces diverses approches tournent toutes autour de l'idée de médiance, et toutes témoignent que la conception de l'humain comme un simple individu n'est plus soutenable. Plus que tout autre vivant - car il a, infiniment plus que toute autre espèce, prothésé son corps de systèmes techniques et symboliques, tous nécessairement collectifs et constitutifs de son existence même -, il est au contraire médial ; c'est-àdire qu'il ne peut vivre sans un corps médial éco-techno-symbolique, couplé à son corps individuel, et qui est son milieu.

Notre ontologie, néanmoins, reste largement celle du dualisme et de l'individualisme qui lui est corrélatif. Nous sommes encore loin d'accepter facilement l'idée que la réalité qui nous entoure n'est pas celle d'un environnement (Umgebung) objectal (constitué d'objets), toisé par un sujet individuel, mais celle d'un milieu (Umwelt) où les choses participent de notre être même du fait de notre médiance. Le Dasein heideggérien lui-même a peu modifié la donne; car, comme le remarque subtilement Watsuji, l'idée que celui-ci serait un " être vers la mort» (Sein zum Tode) est essentiellement individualiste. La 
médiance, elle, entraîne que l'humain est au contraire un " être vers la vie " (sei e no sonzai 生への存在) ${ }^{7}$, car le corps médial survit au corps animal.

La mésologie ${ }^{8}$ (füdogaku 風土学 ou füdoron 風土論), en revanche, a développé à cet égard un appareil conceptuel rendant spécifiquement compte de ce " troisième et autre genre ", entre sujet et objet, qui est celui de la réalité des milieux humains, et qui découle de notre médiance. Les choses en effet participent de notre corps médial, i.e. de notre milieu. Elles ne sont pas seulement physiques, mais éco-techno-symboliques. C'est ce dont rend compte le concept de trajectivité (Berque 1986) : entre les pôles théoriques du sujet et de l'objet, du pour-soi et de l'en-soi, les choses sont trajectives.

La trajectivité signifie que les choses existent en fonction de la manière dont nous les saisissons par les sens, par la pensée, par les mots et par l'action. Cette saisie, ou prise, est analogue à une prédication dont le sujet $S$ est l'en-soi de la chose en tant qu'objet (i.e. l'Umgebung), et le prédicat $P$ la manière dont la chose nous apparaît du fait de cette prise. La réalité $r$ de la chose (i.e. l'Umwelt) peut donc s'exprimer par la formule $r=S / P$; ce qui se lit : la réalité, c'est $S$ en tant que $P$.

Il s'agit là d'autre chose que d'une projection subjective sur l'objet. La trajectivité résulte d'un processus historique, la trajection, où il y a indéfiniment subjectivation de l'objet, objectivation du sujet, et transmission cumulative de ce changement. Chaque nouvelle génération prend en effet comme un donné (une Gebung), i.e. comme $S$, ce qui en réalité a été construit $(\mathrm{S} / \mathrm{P})$ par les générations précédentes, et le saisit à neuf par de nouvelles prises. Ainsi, le donné initial (S) ne cesse d'évoluer en $S^{\prime}, S^{\prime}$, $S^{\prime \prime}$, etc., pour être indéfiniment prédiqué en P', P", P'”, etc. Autrement dit, il y a indéfiniment assomption de $S$ en $P$, et hypostase (substantialisation) $)^{9}$ de $P$ en $S^{\prime}$; ce qui peut se rendre par la formule suivante : ((S/P)/P')/P”)/P”'...

Cela signifie non seulement que l'humain n'est jamais, comme le cogito du dualisme, face à de simples objets là-devant, mais qu'il est lui-même indéfiniment construit par son milieu, qu'il construit lui-même ; construction

7. Sur ce thème, voir Berque et al. 2008.

8. Sur ce qui suit, voir Berque 2009, 2010, 2011.

9. Rappelons que « sujet" (subjectum, traduisant hupokeimenon) et "substance " (substantia, traduisant hupostasis) relèvent d'une même image : celle de ce qui " gît (keimai) dessous, se tient (stasis) dessous ", i.e. de fondement des choses, et à propos de quoi se dit quelque chose (P). Pour Aristote, P n'est donc pas substantiel. 
mutuelle, ou concrescence (croître-ensemble), dont l'état présent n'est autre que la médiance.

Effectivement, ce " moment structurel de l'existence humaine " qu'est la médiance ne cesse d'évoluer au cours de l'histoire ; et cela indéfiniment sous le signe de la contingence, puisque le choix de P dépend toujours de l'existant lui-même. En revanche, cette contingence n'a rien à voir avec l'arbitraire d'une simple projection du cogito sur l'en-soi d'un objet, puisque la chose est toujours déjà construite $(\mathrm{S} / \mathrm{P}$ et non pas $\mathrm{S})$ et que l'existant humain est nécessairement médial, c'est-à-dire lui-même toujours déjà construit dans ce rapport ; ce qui entraîne que, dans cette histoire, il n'y a ni hasard ni nécessité, mais bien ce troisième et autre genre qu'est la contingence. En effet, dans la médiance et la trajectivité de l'histoire et du milieu, les choses auraient toujours pu être autrement qu'elles ne sont; mais elles sont ce qu'elles sont $(\mathrm{S} / \mathrm{P})$ en fonction de l'histoire d'un certain milieu : la leur, toujours singulière.

Le même principe s'applique à l'échelle ontologique du vivant en général ; et particulièrement à ce qu'on appelle l'évolution ${ }^{10}$.

\section{Logos et lemme}

Si la perspective ontologique ainsi ouverte par la mésologie semble bien avoir dépassé le dualisme moderne, qu'en est-il de son armature logique ? Qu'en est-il, en particulier, de ce " troisième et autre genre " que le principe du tiers exclu a depuis si longtemps forclos?

La question que l'on entrevoit d'emblée, c'est que les fondements classiques de la rationalité en Occident - les trois principes d'identité, de contradiction et d'exclusion du tiers déjà utilisés par Aristote pour structurer la logique du sujet - ne permettent de saisir ni la médiance, ni la trajection. La solution ne pouvait être de renverser la logique du sujet en logique

10. Nous ne pouvons ici développer cette question. Soulignons seulement la perspective : de telles vues ne concordent pas avec le néo-darwinisme standard, où la question est posée en termes mécanicistes, c'est-à-dire d'Umgebung et non pas d'Umwelt; mais la théorie de l'évolution, du fait de la révolution post-génomique et épigénétique, est en train d'amorcer un profond bouleversement. Voir par exemple Jablonka \& Lambs 2005, et Paldi 2009. 
du prédicat, comme l'a tenté Nishida ; car la seconde, ainsi que l'a bien vu Nakamura Yūjirō 中村雄二郎 (1989 : 194), revient aux métaphores d'une "paléologique " (le terme est du psychanaliste Sylvano Arieti), qui certes est à l'œuvre dans toute pensée humaine et que par conséquent, serait-ce en termes d'inconscient, nous devons reconnaître, mais qui ne peut certainement pas remplacer la logique du sujet pour ce qui est du rationnel.

Nishida, cependant, a mis le doigt sur un point décisif pour la mésologie : le monde est prédicatif (jutsugo sekai 述語世界). Voir ainsi les choses permet notamment de comprendre que cet obscur " litige " (Streit) que Heidegger, dans l'Origine de l'œuvre d'art (1962: 53), imagine entre la terre et le monde ${ }^{11}$, n'est autre que le rapport entre $S$ (la terre ou l'Umgebung, sujet premier de toute la prédication humaine) et $\mathrm{P}$ (le monde ou l'Umwelt, c'est-à-dire l'ensemble des prédicats humains) ; autrement dit, c'est la réalité S/P, dans laquelle le « litige » correspond à la trajection de $S$ en P. Litige effectivement il y a, puisque ce rapport n'est pas réductible à l'identité : c'est un "moment ", toujours dynamique.

Mais quelle est donc la nature logique des " en-tant-que ${ }^{12}$ ", ou prises, que nous avons sur cette réalité ? Ces prises que nous offrent les choses concrètes de notre milieu, en effet, ne sont pas réductibles à l'identité de l'objet (autrement dit $S$ ) ; mais elles ne le sont pas non plus à celle du prédicat (la manière de saisir $S$ ), puisque comme on l'a vu, dans la trajection, il y a indéfiniment assomption de $\mathrm{S}$ en $\mathrm{P}, \mathrm{P}$ ', P”, etc., et hypostase de $\mathrm{P}$ en S', S", S", etc.

Or c'est bien, hormis le concept lui-même, cette trajection qu'éclaire la " logique du lemme » (renma no ronri レンマの論理) - disons carrément la lemmique, puisque ce n'est justement pas une « logique» au sens de logique du logos, rogosu no ronriロゴスの論理 - que Yamauchi a mise en avant dans Logos et Lemme. La logique du logos s'en tient aux trois principes d'identité, de contradiction et du tiers exclu. C'est le cas même dans la dialectique hégélienne, où la synthèse ne fait pas coexister la thèse et l'antithèse, mais

11. Mon interprétation est plus explicitement argumentée dans Berque 2009, Chap. V. 12. Cette question de l'en-tant-que a été posée par Heidegger dans son cours de 1929, en rapport justement avec la théorie des milieux d'Uexküll ; mais ce cours n'ayant été publié qu'en 1983, sous le titre Grundbegriffe der Metaphysik. Welt-EndlichkeitEinsamkeit (Heidegger 1992), soit après la mort des deux philosophes, Yamauchi n'en avait pas connaissance. 
les sursume (aufhebt, c'est-à-dire les abolit et les surélève à la fois), respectant donc le principe du tiers exclu ${ }^{13}$.

Yamauchi montre que cette impossibilité d'admettre le tiers (ce qui est à la fois $\mathrm{A}$ et non-A) découle du déploiement même du logos (Chap. II, Rogosu no tenkai ロゴスの展開), qui a conduit celui-ci à se poser en luimême, dans une logique formelle abstraite, i.e. découplée des choses et face à des objets. La pensée orientale en revanche, dans le bouddhisme en particulier, s'est gardée d'absolutiser ainsi la parole (logos). C'est ce qu'illustre le tétralemme nagarjunien (catuşkoți, shiku 四句 ou shiku bunbetsu 四句 分別), qui déconstruit toute "logique " par une suite de quatre lemmes : $\mathrm{A}$ (assertion), non-A (négation), ni $\mathrm{A}$ ni non-A (binégation), à la fois $\mathrm{A}$ et non-A (biassertion). Seuls le premier et le second de ces lemmes sont admissibles dans la « logique ", où ils correspondent aux principes d'identité et de contradiction. Le troisième et le quatrième lemme, qui transcendent ces principes, correspondent à ce que le bouddhisme du Grand Véhicule appelle "vérité suprême ", paramārtha (shōgi 勝義).

Contrairement à la plupart des commentateurs en la matière ${ }^{14}$, Yamauchi place la binégation (ni A ni non-A) non pas en quatrième, mais en troisième position. Il montre en effet que celle-ci est le nœud du tétralemme, car c'est elle qui non seulement rend possible la biassertion (à la fois A et non-A), mais en fait un véritable déploiement ; sinon, en effet, le tétralemme répéterait indéfiniment les mêmes boucles.

Il n'y a pas lieu ici d'entrer dans le détail d'un livre de 400 pages sur un pareil thème. L'idée centrale de Yamauchi est que le tétralemme permet de dépasser les limitations du logos. Il y a là beaucoup à prendre du point de vue de la mésologie, comme nous le verrons plus loin ; mais posons d'emblée une frontière. C'est que, pour Yamauchi, les deux derniers lemmes expriment bien une vérité suprême : un absolu. En cela, sa lemmique

13. La découverte de l'intrication quantique a cependant conduit certains penseurs européens, comme Stéphane Lupasco, a imaginer une logique admettant le tiers (à la fois A et non-A). Sur ce point, v. Nicolescu 2009. Nous nous en tenons ici à la thèse de Yamauchi.

14. Tel Nef 2011, et tous les auteurs commentés dans cet ouvrage. Pour Nagarjuna, l'ordre des deux derniers lemmes paraît assez fluctuant, mais selon Yamauchi, c'est lui néanmoins qui a établi le véritable raisonnement tétralemmique, avec la biassertion en quatrième position. 
adopte telle quelle la position du bouddhisme, qui est celle d'une religion. Il y a là un bond mystique auquel se refuse la mésologie. Pour celle-ci, absolutiser quelque connaissance que ce soit est hors de question; car le fait même de connaître $S$ l'établit dans la perspective $S / P$ d'un certain prédicat $\mathrm{P}^{15}$, serait-il totalement non verbal (telle l'ineffabilité de l'extase mystique ou du satori 悟り dans le zen). Pour ce qui suit, nous nous en tiendrons donc modestement à un usage profane et agnostique du tétralemme, c'est-à-dire à poser la réalité comme $\mathrm{S} / \mathrm{P}$.

\section{Conclusion : la méso-logique est une lemmique}

Les prises éco-techno-symboliques que nous offre notre milieu ne concernent pas seulement le langage (comme la prédication au sens strict), mais encore, et simultanément, les sens, la pensée et l'action. Elles sont donc irréductibles à l'abstraction du logos, comme le manifeste en particulier leur symbolicité. Dans le symbole en effet, A est toujours également non-A (quatrième lemme). Plus largement, l'en-soi des choses (ce qui serait la pure identité de $S$ ) ne nous étant accessible que comme $S / P$, la réalité dépasse nécessairement le principe d'identité : $S$ suppose $P$, qui suppose $S$. Il y a là, entre $S$ et $\mathrm{P}$, non seulement relation (sōtai 相対), mais interdépendance. Il y a même, dans la concrescence historico-médiale des choses et des existants, ce que Yamauchi, reprenant un terme bouddhique, a nommé sōdai (相待) : coattente ${ }^{16}$.

15. Ce à quoi correspond, en physique quantique, le principe d'indétermination de Heisenberg.

16. 相待 (ch. xiangdai, jap. södai) est la traduction que le bouddhisme chinois a donnée du sanskrit apekșa (idée de prendre en compte), en empruntant ce terme au Zhuangzi, qui l'utilise dans le Qiwulun 斉物論 ("De la mise à plat qui rend les choses équivalentes " selon la traduction proposée par Anne Cheng (1997 : 111), où le terme se trouve dans ce passage (II, 34) : hua sheng zhi xiang dai, ruo qi bu xiang dai 化聲之相待、 若其不相待, «les oppositions discursives ne sont qu'absence d'opposition ». Autrement dit, les oppositions se nourrissent réciproquement à partir de l'absence d'opposition. En deçà de toute distinction et division introduite par le langage, gît un fond indistinct et indivis. 
La coattentivité (sōdaisei 相待性) est l'un des concepts les plus forts de Logos et Lemme. On peut utilement le rapprocher de celui de médiance : entre corps individuel et corps médial, il y a effectivement coattente. C'est bien ce que, sans rien connaître de ces concepts ni de leurs auteurs, LeroiGourhan a montré par son interprétation de l'émergence de notre espèce ; théorie que l'on peut résumer de la façon suivante : au cours de ce processus, se sont répondus - autrement dit coattendus - l'anthropisation du milieu par la technique, l'humanisation du milieu par le symbole, et l'hominisation du corps animal.

C'est au demeurant l'idée même de lemme qui éclaire la mésologie. Comme on l'a vu, ce terme dérive d'un verbe qui veut dire " prendre ». Les prises médiales relèvent bien d'une lemmique, antécédente et englobante par rapport à la " logique » (ce qui est la thèse de Yamauchi). Elles fonctionnent en effet bien en deçà de notre conscience, et donc de tout rationalisme. Comptant inconsciemment sur ces prises, nous les prenons comme un donné (une Gebung, un taken for granted allant de soi), autrement dit comme un $S$ alors que c'est notre propre histoire (et en deçà, l'évolution de notre espèce) qui les a élaborées en $S / P$, pour les hypostasier indéfiniment en S', S" etc. Ces hypostases - ces substantialisations en $S$ de l'insubstantiel $\mathrm{P}$ - correspondent à ce que Yamauchi, reprenant un autre terme bouddhique, nomme eji 依止 (également lu eshi), traduction du sanscrit niśraya. Girard (2008) rend ce terme par " appui ", et cite cet exemple éclairant ${ }^{17}$ : "C'est parce qu'ils sont sans nature propre que [tous les dharma] s'érigent/ L'antérieur est le point d'appui du postérieur (Wu ziti gu cheng/Qian wei hou yizhi 無自体故成、前為後依止) 》.

Effectivement, dans les milieux humains, comme dans les milieux vivants en général, la réalité est sans nature propre (ce n'est pas l'en-soi d'un objet), puisqu'elle résulte d'une trajection : l'assomption de S en P, l'hypostase $\mathrm{P}$ en $\mathrm{S}^{\prime}$, et ainsi de suite. Néanmoins, nous comptons sur ses prises $(\mathrm{S} / \mathrm{P})$; autrement dit sur les appuis qu'elle offre à notre existence, par calage trajectif (l'hypostase de P en S'). La différence entre ces cales et les eji du bouddhisme, c'est que, comme le montre Yamauchi, ces derniers relèvent en dernière instance de l'absoluité (zettaisei 絶対性) ou, préfère-t-il dire, de l'abtentivité (zetsudaisei 絶待性) du vide ( $k \bar{u}$ 空), alors que la mésologie ne

17. Qu'il tire du Mahāyānasūtrālamkāra, XI-51, t. XXXI, n 1604, p. 615a et 623a. 
peut accomplir le bond mystique qui mènerait au-delà du rapport $S / P$ : elle supposera toujours que $P$ se cale sur $S$, celui-ci serait-il à jamais un S', c'està-dire toujours déjà $S / P$. L'absoluité en moins, cela revient tout de même à dire ce que dit le troisième lemme, celui de la binégation ; car S/P n'est ni $S$, ni $\mathrm{P}$, mais toujours déjà leur trajection. Et cela revient aussi à dire ce que dit Yamauchi en plaçant ce troisième lemme avant le quatrième (la biassertion) ; car c'est bien la double négation de $S$ et de P qui permet le dépassement de leur identité respective, donc la concrescence de la réalité $S / P$, où trajectivement, et définitivement au-delà de la modernité, se coattendent les mots et les choses, les choses et notre existence, tout cela produisant historiquement ce " troisième genre " qu'est la médiance de notre milieu : l'empreinte et la matrice à la fois (quatrième lemme) de notre genesis.

\section{Références}

AGAMBEN Giorgio, 2002

L'Ouvert: de l'homme et de l'animal, Paris, Payot \& Rivages.

BERQUE Augustin, 1986

Le Sauvage et l'Artifice. Les Japonais devant la nature, Paris, Gallimard.

BERQUE Augustin (dir.), 2000

Logique du lieu et dépassement de la modernité, Bruxelles, Ousia, 2 vol.

BERQUE Augustin, 2002

«La logique du lieu dépasse-t-elle la modernité ? ", in MONNET Livia (dir.), Approches critiques de la pensée japonaise au $x x^{e}$ siècle, Montréal, Presses de l'université de Montréal, pp. 41-52.

\section{BERQUE Augustin, 2002}

«Du prédicat sans base : entre mundus et baburu, la modernité ", in MONNET Livia (dir.), Approches critiques de la pensée japonaise au $x x^{e}$ siècle, Montréal, Presses de l'université de Montréal, pp. 53-62.

BERQUE Augustin, 2009 [or. 2000] Écoumène. Introduction à l'étude des milieux humains, Paris, Belin.

BERQUE Augustin, 2010 Histoire de l'habitat idéal. De l'Orient vers I'Occident, Paris, Le Félin.

BERQUE Augustin, 2011

Milieu et identité humaine. Notes pour 
un dépassement de la modernité, Paris, Donner lieu.

\section{BERQUE Augustin, 2012}

"La chôra chez Platon », in PAQUOT Thierry et YOUNÈS Chris (dir.), Espace et lieu dans la pensée occidentale de Platon à Nietzche, Paris, La Découverte, pp. 13-27.

\section{BERQUE et al., 2008}

"Être vers la vie. Actes du colloque de Cerisy », Ebisu, 40-41 : 9-224.

\section{BOI Luciano, 2008}

« Epigenetic Phenomena, Chromatin Dynamics, and Gene Expression. New Theoretical Approaches in the Study of Living Systems », Rivista di Biologia/ Biology Forum, 101 (3) : 405-442.

\section{BRISSON Luc, 1994}

Le même et l'autre dans la structure ontologique du Timée de Platon,

Sankt Augustin, Academia Verlag.

CANGUILHEM Georges, 2002 [or. 1968] Études d'histoire et de philosophie des sciences concernant les vivants et la vie, Paris, Vrin.

CANGUILHEM Georges, 2009 [or. 1965] La Connaissance de la vie, Paris, Vrin.

\section{CHENG Anne, 1997}

Histoire de la pensée chinoise, Paris, Seuil.

\section{DUFOUR Dany-Robert, 2005}

On achève bien les hommes. De quelques conséquences actuelles et futures de la mort de Dieu, Paris, Denoël.

FÈBVRE Lucien, 1949 [or. 1922]

La Terre et l'évolution humaine, Paris, Albin Michel.

\section{GIRARD Frédéric, 2008}

Vocabulaire du bouddhisme japonais, Genève, Droz, 2 vol.

HEIDEGGER Martin, 1962 [or. 1949] Chemins qui ne mènent nulle part, Paris, Gallimard.

HEIDEGGER Martin, 1992 [or. 1983]

Les concepts fondamentaux de la métaphysique. Monde-finitude-solitude, Paris, Gallimard.

\section{JABLONKA Eva}

\& LAMBS Marion J., 2005

Evolution in Four Dimensions, Cambridge MA, MIT Press.

\section{LEROI-GOURHAN André, 1964}

Le Geste et la Parole, Paris, Albin Michel, 2 vol.

\section{MERLEAU-PONTY Maurice, 1945}

Phénoménologie de la perception, Paris, Gallimard.

NAKAMURA Yūjirō 中村雄二郎, 1989 Basho (toposu) 場所(トポス) (Lieu), Tokyo, Kōbundō 弘文堂.

\section{NEF Frédéric, 2011}

La force du vide. Essai de métaphysique, Paris, Seuil. 
NICOLESCU Basarab, 2009

Qu'est-ce que la réalité ? Réflexions

autour de l'œuvre de Stéphane Lupasco,

Montréal, Liber.

PALDI Andràs, 2009

L'Hérédité sans gènes, Paris, Le Pommier.

UEXKÜLL Jakob von, 2010 [or. 1934]

Milieu animal et milieu humain, Paris,

Payot \& Rivages.

WATSUJI Tetsurō, 2011 [or. 1935]

Fūdo. Le milieu humain, Paris, CNRS.

YAMAUCHI Tokuryū 山内得立, 1974

Rogosu to Renma ロゴスとレンマ

(Logos et Lemme), Tokyo, Iwanami

shoten 岩波書店. 